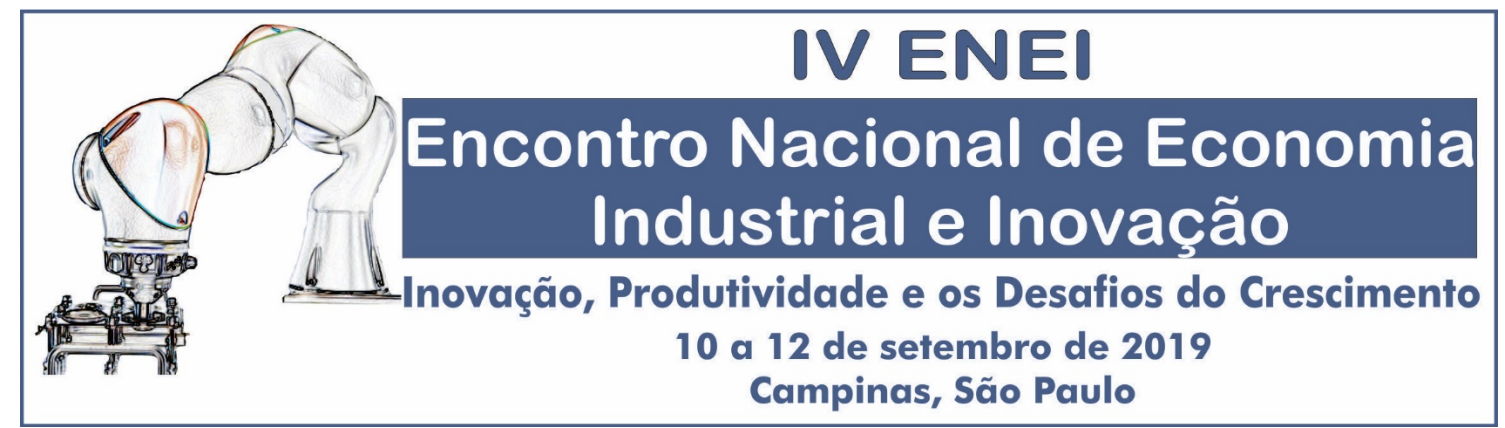

\title{
FONTES DE INFORMAÇÃO PARA INOVAÇÃO DA INDÚSTRIA BRASILEIRA: UMA ANÁLISE A PARTIR DE MEDIDAS DE CENTRALIDADE DE GRAFOS.
}

\author{
Bruno Ferreira de Oliveira ${ }^{1}$ \\ Felipe Ponciano da $\mathrm{Cruz}^{2}$ \\ Enzo Matheus Fernandez Barreira Accioly ${ }^{3}$
}

\begin{abstract}
Resumo
O objetivo do artigo é mapear a estrutura das fontes de informação para inovação da indústria brasileira listando os setores industriais mais dinâmicos e a relevância de cada fonte para o processo inovativo, utilizando medidas de centralidades de grafos, a partir da base de dados da PINTEC de 2008, 2011 e 2014. Os resultados indicam que os segmentos industriais que buscam de forma mais intensa as fontes de informação são os de baixa e média-baixa intensidade tecnológica. Em relação à relevância das fontes de informação, constatou-se um padrão de concentração das fontes utilizadas, relacionadas com os clientes ou consumidores, redes de informações informatizadas, fornecedores, feiras e exposições e concorrentes, evidenciando o aspecto incremental e imitativo dos projetos de inovação. Além disso, verifica-se a baixa representatividade das universidades e institutos de pesquisa como fontes de informação relevantes para implementação das inovações na indústria brasileira.
\end{abstract}

Palavras-chave: Fontes de informação; inovação; medidas de centralidades de grafos; indústria brasileira.

\begin{abstract}
The aim of the article is to map the sources of information for innovation of the brazilian industry listing the most dynamic sectors and sources of information, using as instrument measures of centrality of graphs, from the database of PINTEC of 2008, 2011 and 2014. The results are that the industrial segments that seek greater intensity as sources of information are from low and medium-intensity technology. In relation to the relevance of the information sources, it is a pattern of concentration of the sources used, related to customers or consumers, computerized information networks, suppliers, fairs and exhibitions and groups, showing the incremental and imitative aspect of innovation projects. In addition, it verifies the low representativeness of universities and research institutes as sources of information relevant to the implementation of innovations in brazilian industry.
\end{abstract}

Keywords: Sources of information; innovation; measures of centrality of graphs; brazilian industry.

Área 5.4: Economia do Conhecimento

JEL: O31; O32

\footnotetext{
${ }^{1}$ Mestrando em Economia pela Universidade Cândido Mendes (UCAM)

${ }^{2}$ Mestre em Economia Aplicada da Universidade do Estado do Rio de Janeiro (UERJ)

${ }^{3}$ Graduando em Economia pela Universidade Federal do Rio de Janeiro (UFRJ)
} 


\section{Introdução}

O conhecimento da dinâmica capitalista sob a forma evolutiva é fundamental para entender a maneira como se desenvolvem novos produtos, processos, novos mercados e novas formas de organização industrial. Dessa forma, evidencia-se a importância do processo inovativo como um dos vetores de relevância para o desenvolvimento econômico de um país. No contexto de um mundo globalizado e com o acirramento da competição em nível internacional, a inovação é destacada como fator crucial para a determinação do padrão de concorrência e a competitividade setorial (DOSI; MALERBA; ORSENIGO, 1994; FERRAZ; KUPFER; HAGUENAUER, 1995).

Nessa perspectiva, a dinâmica econômica está relacionada com a capacidade de aprendizado dos agentes, ou seja, na forma como os agentes econômicos destroem (esquecem) os elementos antigos e criam elementos novos de forma contínua e crescente. Assim, pode-se definir a inovação como sendo a utilização do conhecimento sobre novas formas de produzir e comercializar bens e serviços (LASTRES; FERRAZ, 1999).

O sucesso e sustentabilidade das organizações está relacionado a sua capacidade de diferenciação frente aos seus concorrentes, bem como envidar esforços para estar na vanguarda tecnológica no setor que atua. Neste cenário, a busca pelas diversas fontes de informação existentes por parte das firmas é alvo vital para orientar seus esforços de inovação, construindo capacitações e habilidades relevantes para a sua atividade.

Segundo dados da Pesquisa de Inovação Tecnológica de 2014 (PINTEC), elaborado pelo Instituto Brasileiro de Geografia e Estatística (IBGE), a taxa de inovação da indústria é de 36,4\%, referente ao triênio 2012-2014, onde uma parte relevante das empresas declararam que as inovações implementadas estão relacionadas a novo processo, além das atividades inovativas estarem relacionadas, em boa parte, com aquisição de máquinas e equipamentos e treinamento (IBGE, 2016).

Assim, como as fontes de informações são cruciais no processo inovativo, o objetivo deste artigo é mapear por meio da análise das medidas de centralidade de grafos, a estrutura de fontes de informação para o processo inovativo da indústria brasileira, listando os setores industriais mais relevantes na busca das referidas fontes de informação, bem como mensurar quais fontes de informação são mais relevantes para o processo inovativo da indústria brasileira.

Para viabilizar a análise, foram utilizados os dados das empresas que implementaram inovações, com a consideração do grau de importância listada pela PINTEC, elaborada pelo IBGE, para os períodos de 2008,2011 e 2014.

O artigo é divido em cinco seções: após a seção introdutória segue o referencial teórico sobre o processo de aprendizado e construção de capacitação tecnológica das organizações, a terceira seção trata dos aspectos metodológicos da pesquisa, os resultados são expostos na quarta parte e, por fim, segue a conclusão.

\section{Referencial Teórico: Aprendizado e fontes de informação}

A compreensão da economia do aprendizado, relacionado com a importância da informação e do conhecimento como forma de construção de vantagens competitivas para as empresas, deflagra o papel da inovação como forma de expressão do desenvolvimento, se expressando na compreensão dos sistemas de inovação, sejam eles nacionais, regionais ou setoriais (FREEMAN, 1995; MALERBA, 2002; DOSI; MALERBA; ORSENIGO, 1994).

Segundo Freeman (1995), as firmas inovadoras em seus países foram aquelas que firmaram redes de contatos, de forma a acumular capacitação e aprendizagem em conjunto. Efeitos estes que fizeram os países de industrialização tardia darem um salto tecnológico relevante fundamental para o seu desenvolvimento.

A base do sistema nacional de inovação está pautada em algumas características: (i) Baseado em educação e processo de treinamento (geração do conhecimento); (ii) Baseado em ciência; (iii) Institutos tecnológicos; (iv) Acumulação de conhecimento; (v) Promoção de estratégias industriais; (vi) Adaptação da tecnologia importada (processo de imitação); e (vii) Aprendizado interativo.

A partir dos anos 1980, observa-se que, aliado à conjuntura econômica global, houve um processo de incentivo e acirramento da competitividade em nível internacional com ênfase na inovação. Neste 
momento, o foco da firma muda de planejamento da produção para uma visão estratégica da firma como um todo, onde segundo Freeman (1995), o mais importante é monitorar e interpretar as implicações das mudanças tecnológicas e dos mercados e adaptar sua capacitação interna para responder a esta mudança na estrutura tecnológica, que segundo Dosi (2006) é caracterizado pela trajetória no qual a firma e/ou a indústria está inserida. Desta forma, a visão estratégica está pautada na capacitação e aprendizado, de forma a oferecer vantagens competitivas à firma no mercado.

Assim, para que um setor possua capacitação e aprendizagem para gerar a inovação, ele tem que dominar os processos básicos e estudá-los, assim o conhecimento e a informação são elementos crucias na atividade econômica. A forma atual de desenvolver a economia coloca opções amplas e inesgotáveis de codificação de conhecimento para a melhoria do bem-estar da sociedade.

Segundo Johnson e Lundvall (2005), a economia baseada no processo de conhecimento e aprendizado está relacionado com a capacidade de renovação do estoque de conhecimento e competências de forma acelerada e contínua. Assim, as organizações que possuem elevada capacidade de aprendizagem, tem chances maiores de sobrevivência num ambiente de competição hostil.

Desse modo, define-se o aprendizado como o desenvolvimento de novas competências e construção de novas capacitações e habilidades. Vale ressaltar que nesse contexto, o acesso a novas informações não é sinônimo de aprendizagem, porém é o insumo para a construção de habilidades e competências.

Malerba (1992) define o processo de aprendizagem como um processo custoso para as organizações, relacionado com as fontes internas e externas de conhecimento, resultando em melhorias no estoque de conhecimento e nas suas capacidades tecnológicas, conformando na construção de trajetórias de avanço tecnológico.

Outro conceito de aprendizado apresentado na literatura o define como "um processo pelo qual a repetição e a experimentação permitem que as tarefas sejam mais bem e mais rapidamente desempenhadas e que as novas oportunidades de produção sejam identificadas" (TEECE, 2005, p.154)

$\mathrm{Na}$ economia do aprendizado, a inovação é um processo interativo de natureza social e coletiva (EDQUIST; LUNDVALL, 1993), que impulsiona as relações e interações entre os agentes, de acordo com o grau de especificidades do nível de conhecimento e do escopo tecnológico demandado pelo setor (MALERBA, 2004).

Uma das características relevantes do processo de aprendizagem vai além da prática da imitação, sendo relevante a construção em conjunto de contribuições e entendimentos para problemas de elevado grau de complexidade (TEECE, 2005).

Neste sentido, o processo de aprendizagem pode ser associado a busca de diferenciação da firma de seus concorrentes. Para a teoria evolucionária, "a firma individual é encarada como um repositório de conhecimentos produtivo e tecnológico constantemente em busca de soluções mais vantajosas entre as diversas possíveis" (Bittencourt, 2012, p. 39).

Para Lazonick (2000) a firma pode ser entendida como organizações que representam o conhecimento social de coordenação e aprendizado. Aliado ao processo de aprendizagem, temos a importância do conhecimento, sendo este idiossincrático em cada firma, além da necessidade de sua absorção pelas organizações por meio de habilidades acumuladas no tempo, dado que o conhecimento não é difundido de forma automática (MALERBA, 2002).

O conhecimento está relacionado com a construção de capacidade dinâmica das organizações, relacionadas com as competências internas e externas, como forma de resposta à rapidez das mudanças do ambiente competitivo pela qual estão sujeitas (TEECE; PISANO; SHUEN, 1997).

As formas mais comuns de aprendizado que as firmas estão sujeitas - sejam fontes internas ou externas - apresentadas pela literatura como fontes de construção de conhecimento são: (i) Learning by doing - relacionada com a atividade produtiva da organização; (ii) Learning by using - relacionada com o uso de produtos, maquinário e insumos de produção; (iii) Learning from advances in science and technology - atividade externa a organização e relacionada a absorção de novas técnicas relacionadas à ciência e tecnologia; (iv) Learning from inter-industry spillovers - atividade externa a organização e relacionada com as técnicas produtivas do concorrente; (v) Learning by interacting - atividade externa a organização e resultante de interação entre consumidores e fornecedores ou o estabelecimento de 
cooperação com outras empresas do setor; e (vi) Learning by searching - atividade interna à organização, relacionada na construção de novos conhecimentos por meio da área de P\&D (MALERBA, 1992).

Esses mecanismos de aprendizado e fontes de conhecimento geram impactos na estrutura tecnológica por meio de geração de novos processos de produção, alterações de insumos usados no processo produtivo, alterações na escala e na organização do processo produtivo, além da diferenciação horizontal e/ou vertical do produto (MALERBA, 1992).

Para subsidiar o presente estudo, realizou-se uma revisão bibliográfica de artigos empíricos relacionados com o tema no Brasil. Com relação a análise das fontes de inovação no Brasil, Gonçalves e Simões (2005) analisaram os componentes principais e de cluster (análise multivariada) sobre um determinado conjunto de indicadores da PINTEC, nos anos de 1998 e 2000, sendo os resultados contrários aos esperados na literatura para os setores "dominados por fornecedores" e os setores baseados em esforços de $P \& D$, principalmente os setores intensivos em escala. Os setores com oportunidades tecnológicas relevantes possuem gastos em P\&D modestos em comparação aos seus desafios.

Campos e Ruiz (2009) estudam os padrões de inovações dos setores da indústria brasileira com o objetivo de identificar o comportamento e estrutura dos setores da indústria. Os autores utilizam a análise de clusters hierárquicas e não-hierárquicas como recurso metodológico e empregam cinco indicadores para retratar a inovação industrial, como "origem da inovação", "principal fonte inovativa", "aprendizagem predominante", "foco da trajetória tecnológica" e "resultados inovativos", sendo utilizados dados da PINTEC do ano de 2000. Os resultados encontrados indicam uma marcante heterogeneidade de características de inovação na indústria brasileira, indicando trajetórias diferenciadas para cada setor. Para fins de política industrial inovativa, o resultado implica que políticas industriais lineares para os diversos setores não se tornam eficazes para gerar impacto no processo inovativo. Uma política inovativa nessa perspectiva deve abranger o reconhecimento da heterogeneidade dos diversos setores.

Silva e Suzigan (2014) investigam os padrões setoriais de inovação da indústria de transformação brasileira por meio da análise de cluster. Para realização da análise os autores utilizaram dados da PINTEC de 2008 produzidos pela Pesquisa Industrial Anual (PIA) da Secretária do Comércio Exterior (SECEX). Foram selecionados 53 indicadores, agrupados em 7 categorias, buscando capturar as seguintes informações dos setores industriais: (i) estrutura e desempenho, (ii) resultados, (iii) esforços, (iv) fontes de informação, (v) fontes de inovação, (vi) interatividade, (vii) foco da trajetória tecnológica. Os resultados encontrados demonstraram aderência majoritária dos setores com os resultados esperados da literatura.

Bittencourt (2012) utiliza a técnica de clustering de análise estatística multivariada para identificar padrões setoriais de aprendizagem na indústria brasileira, com dados da PINTEC para os anos 2000, 2003 e 2005. O resultado apresentado pelo referido trabalho é a divisão dos setores industriais em quatro padrões industriais de aprendizagem, como intensivo em aprendizagem na esfera produtiva, intensivo em aprendizagem à montante, intensivo em múltiplas formas, intensivo em aprendizagem interna e à jusante.

Bittencourt, Britto e Giglio (2016) construíram indicadores de tipos de aprendizagem e graus de inovação para compreensão da dinâmica inovativa da indústria brasileira, por meio do método de mínimos quadrados generalizados. Os resultados encontrados são que inovações mais complexas decorrem de formas de aprendizagem que combinam conhecimentos codificados e tácitos, evidenciando a heterogeneidade nas fontes de informação, ao passo que inovações menos complexas dependem apenas de conhecimentos tácitos, além de evidenciar que a indústria brasileira concentra nos clientes como principal fonte de informação para inovar.

Alternativamente à metodologia de análise apresentada pelos trabalhos listados, este artigo mapeará a partir da teoria de grafos e análise de redes a intensidade de busca de informações dos setores industriais comparado com a taxonomia de intensidade tecnológica da Organização para a Cooperação e Desenvolvimento Econômico (OECD, 2011) e Pavitt (1984) por meio da sistematização proposta para a indústria brasileira por Cavalcante (2014), bem como as principais fontes de informação para o processo inovativo da indústria brasileira, a luz do referencial teórico adotado.

\section{Aspectos Metodológicos}

\subsection{Medidas de Centralidade em Grafos}


O interesse crescente pelo estudo de redes pela comunidade científica deve-se a capacidade de modelagem de situações relacionadas com o estabelecimento de relações sociais, associada com a sua utilização para analisar as interações entre os agentes.

Assim, segundo De Freitas (2010), a rede pode ser descrita matematicamente por meio de grafo, utilizado para descrição de sua estrutura topológica. O grafo é um objeto matemático definido por um conjunto finito de elementos denominados vértices e um segundo conjunto cujos elementos são denominados arestas, representando a conexão entre dois vértices (FERREIRA, 2014). Segundo Ferreira (2014), as arestas podem ser múltiplas, com possibilidade de direção definida, bem como a possibilidade de associar números correspondentes a pesos.

Na notação matemática, o grafo denotado por $G(V, E)$ consiste de um conjunto finito não-vazio de $\mathrm{n}$ vértices $v_{i} \in V$, e uma família finita de pares não ordenadas de elementos (não necessariamente distintos) de $\mathrm{m}$ arestas $\left(v_{i}, v_{j}\right) \in E$ (WILSON, 1996; DE FREITAS, 2010). As relações entre os agentes propostos no grafo podem ser colocadas em uma matriz, sendo esta matriz quadrada simétrica, cuja sua ordem seja $n$ $\mathrm{x} \mathrm{n}$.

A matriz que traduz as relações entre os agentes na rede é a Matriz adjacência. A matriz A(G) possui autovalores reais, e dado que o traço da matriz $A(G)$ é igual a zero, então a soma de seus autovalores são zero. O polinômio característico da matriz adjacência $A(G)$ é denotado por det $(\lambda I-A(G))$, onde $\lambda$ é o autovalor do Grafo $G$. Considerando que a matriz $A(G)$ possui autovalores distintos, de forma a obter $\lambda 1 \geq$ $\lambda 2 \geq \lambda 3 \geq \ldots \geq \lambda \mathrm{n}$, temos que o maior autovalor de $\mathrm{A}(\mathrm{G})$ é denominado o índice do grafo $\mathrm{G}$ ou o raio espectral de A.

No caso de análise de redes sociais, os vértices representam os atores e as arestas a relação entre os respectivos atores, sendo importante a identificação dos atores relevantes em relação à rede. Assim, as medidas de centralidade descrevem as propriedades de localização e importância de um agente na rede em questão (BORGATTI, 2005). Assim, a centralidade mede a importância de um agente e sua posição na rede (FREEMAN, 1979; BORGATTI, 2005; BORGATTI; EVERETT, 2005) além de ser um indicador relevante quanto a estrutura das redes.

Quanto às medidas de centralidade, podem-se descrever diversas medidas, no qual incorporam diferentes fatores em sua mensuração ${ }^{4}$, neste artigo, utilizaremos as medidas de centralidade de grau (degree centrality), centralidade de informação (information centrality), e centralidade autovetor (eigenvector centrality)

Segundo Freeman (1979) o grau de um vértice é importante para mensurar a sua atividade de comunicação com os outros vértices da rede em questão. De acordo com De Freitas (2010), a centralidade de grau é a contagem do número de adjacências de um vértice $\mathrm{v}_{\mathrm{i}}$, onde a centralidade de grau é o próprio grau do vértice, onde pode ser denotado por:

$$
C_{D}\left(v_{i}\right)=\sum_{j=1}^{n} a_{i j}, \text { onde } v_{i} \in V
$$

onde $a_{\mathrm{ij}}$ são os elementos da matriz adjacência $A(G)$.

Segundo Borgatti (2005), a outra maneira de interpretar a centralidade de grau é como uma medida de efeitos e influências imediatas, isto é, a capacidade de infectar outros agentes da rede diretamente ou em um tempo especificamente curto.

De acordo com Ferreira (2014), a centralidade de informação mede as posições dos atores quanto à capacidade de receber informação da maior parte do ambiente da rede em questão.

A centralidade de autovetor mensura a importância de um vértice a medida de que o vértice mantenha relações com outros vértices considerados importantes na rede. Mesmo se um vértice estiver conectado apenas com alguns vértices, podendo estes vértices serem considerados importantes, o vértice passa a ser central na rede via relações com outros vértices importantes (KÖNIG; BATTISTON, 2009).

\footnotetext{
${ }^{4}$ Para maiores detalhes sobre as medidas de centralidade utilizadas, consultar Freeman (1979), Bonacich (1987), Borgatti (2005) e Borgatti e Everett (2005)
} 
Segundo Borgatti (2005), a centralidade de autovetor é definido como o principal autovetor, associado ao maior autovalor da matriz adjacência $A(G)$. A equação do autovetor associado é dado por:

$$
A v=\lambda v
$$

Onde $\lambda$ é o autovalor associado (vale destacar que $\lambda$ é constante) a matriz adjacência $A(G)$ e v é o autovetor associado ao autovalor $\lambda$. No cálculo dos autovalores associado a matriz adjacência, o maior autovalor associado irá indicar o cálculo dos autovetores para cada agente da rede, no qual os agentes mais relevantes para a rede possuirão os maiores autovetores associados ao maior autovalor da matriz adjacência em questão.

\subsection{Descrição da Base de Dados e Forma de Construção do Grafo}

Para atender ao objetivo deste trabalho, a base de dados utilizada é a Pesquisa de Inovação Tecnológica (PINTEC), elaborada pelo Instituto Brasileiro de Geografia e Estatística (IBGE), relacionada as pesquisas de 2008, 2011 e 2014.

Cabe salientar que, conforme expostos por IBGE (2016), os dados de natureza qualitativa da referida pesquisa se referem aos triênios de referência. Para simplificar a disposição dos dados neste artigo, o período de 2008 se refere ao triênio de 2006-2008, o período de 2011 se refere ao triênio de 2009-2011 e o período de 2014 se refere ao triênio de 2012-2014.

O grau de detalhamento dos setores industriais segue a Classificação Nacional de Atividade Econômica (CNAE), em sua versão 2.0, sendo os setores industriais analisados de forma agregada em 24 setores conforme quadro abaixo.

Quadro 1: Setores industriais por código CNAE 2.0

\begin{tabular}{|c|c|}
\hline Código CNAE & Setores Industriais \\
\hline 10 & Fabricação de produtos alimentícios \\
\hline 11 & Fabricação de bebidas \\
\hline 12 & Fabricação de produtos do fumo \\
\hline 13 & Fabricação de produtos têxteis \\
\hline 14 & Confecção de artigos do vestuário e acessórios \\
\hline 15 & Preparação de couros e fabricação de artefatos de couro, artigos para viagem \\
\hline 16 & Fabricação de produtos de madeira \\
\hline 17 & Fabricação de celulose, papel e produtos de papel \\
\hline 18 & Impressão e reprodução de gravações \\
\hline 19 & Fabricação de coque, de produtos derivados do petróleo e de biocombustíveis \\
\hline 20 & Fabricação de produtos químicos \\
\hline 21 & Fabricação de produtos farmoquímicos e farmacêuticos \\
\hline 22 & Fabricação de produtos de borracha e de material plástico \\
\hline 23 & Fabricação de produtos de minerais não-metálicos \\
\hline 24 & Metalurgia \\
\hline 25 & Fabricação de produtos de metal, exceto máquinas e equipamentos \\
\hline 26 & Fabricação de equipamentos de informática, produtos eletrônicos e ópticos \\
\hline 27 & Fabricação de máquinas, aparelhos e materiais elétricos \\
\hline 28 & Fabricação de máquinas e equipamentos \\
\hline 29 & Fabricação de veículos automotores, reboques e carrocerias \\
\hline 30 & Fabricação de outros equipamentos de transporte, exceto veículos automotores \\
\hline 31 & Fabricação de móveis \\
\hline 32 & Fabricação de produtos diversos \\
\hline 33 & Manutenção, reparação e instalação de máquinas e equipamentos \\
\hline
\end{tabular}

Fonte: IBGE (2016) 
Utilizou-se para os períodos em questão a tabulação da PINTEC relacionada às empresas que implementaram inovações, por grau de importância das fontes de informação empregada, para a construção do grafo. No Quadro 2, pode-se observar os tipos de fontes de informação para inovação utilizadas pela pesquisa.

\section{PINTEC}

Quadro 2: fontes de informação para inovação e sua respectiva origem, utilizadas pela

\begin{tabular}{|c|c|}
\hline Origem das fontes & Fontes de informação para inovação \\
\hline Interno & P \& D Interno \\
\hline Interno & Outras Áreas \\
\hline Externo & Outra empresa do grupo \\
\hline Externo & Fornecedores \\
\hline Externo & Clientes ou consumidores \\
\hline Externo & Concorrentes \\
\hline Externo & Empresas de consultoria e consultores independentes \\
\hline Externo & Universidades ou outros centros de ensino superior \\
\hline Externo & Institutos de pesquisa ou centros tecnológicos \\
\hline Externo & Centros de capacitação profissional e assistência técnica \\
\hline Externo & Instituições de testes, ensaios e certificações \\
\hline Externo & Conferências, encontros e publicações especializadas \\
\hline Externo & Feiras e exposições \\
\hline Externo & Redes de informação informatizadas \\
\hline
\end{tabular}

Fonte: IBGE (2016).

Cabe salientar que para a seleção da relevância das fontes de informação, adotaremos o mesmo critério utilizado na análise de resultados da PINTEC divulgado pelo IBGE, que é considerar no cálculo apenas a atribuições de média e alta relevância.

Os grafos que serão construídos são direcionais, ou seja, os setores industriais informam quais fontes de informação são relevantes para o processo inovativo. Para a análise proposta, será adotado como metodologia para a construção do grafo a matriz adjacência binária, com estabelecimento de filtro para capturar se a maioria das empresas do setor consideram relevantes determinada fonte de informação para inovar, conforme descrição abaixo:

$$
\overrightarrow{a_{i j}}=\left\{\begin{array}{c}
1, \text { se } \frac{x_{i j}}{X_{i}} \geq 0,5 \\
0, \text { caso contrário }
\end{array}\right\}
$$

Assim, os vértices do grafo serão os setores industriais por CNAE e as fontes de informação para inovação. As arestas vão demonstrar se determinada fonte de informação é considerada média ou alta relevância para o setor, para então, montar o grafo de interação entre as fontes de informação e os setores industriais.

O software UCINET (BORGATTI et al., 2002) foi utilizado para montagem dos grafos e os cálculos das medidas de centralidades expostas na seção metodológica. Vale destacar que a matriz adjacência do grafo de interação entre setores industriais por CNAE e as fontes de informação para inovação é uma matriz simétrica quadrada de ordem $\mathrm{n}=38$. 


\section{Resultados e Discussões}

Como forma de estruturar a apresentação dos resultados, será realizado a segmentação dos resultados por setores industriais e por fontes de informação para inovação, devido à diferenciação da ótica de análise. Tal procedimento foi realizado de forma semelhante em Del-Veccio, Britto e Oliveira (2014) e Hurtado e Mejía (2014)

De forma objetiva, os resultados das medidas de centralidade de grafos por setor industrial denotarão a intensidade pela busca de fontes de informação para inovação, ao passo que as medidas de centralidade para as fontes de informação relacionadas a atividade inovativa evidenciarão a importância de cada uma das fontes para os agentes da rede construída.

Conforme pode-se observar na Figura 1, observa-se que, para o ano de 2008, as fontes de informação que não apresentaram conexões com setores industriais foram "Empresas de consultoria e consultores independentes", "Universidades ou outros centros de ensino superior", "Institutos de pesquisa ou centros tecnológicos", "Instituições de testes, ensaios e certificações" e "Centros de capacitação profissional e assistência técnica".

Para o ano de 2011, verificou-se nesta metodologia que as fontes de informação que não apresentaram conexões com setores industriais foram "Universidades ou outros centros de ensino superior", "Institutos de pesquisa ou centros tecnológicos" e "Centros de capacitação profissional e assistência técnica”, conforme evidenciado na figura 2. No ano de 2014, de acordo com a figura 3, a única fonte de informação que não apresentou conexão com a rede construída foi "Centros de capacitação profissional e assistência técnica".

Figura 1: Estrutura dos Grafos das fontes de informação para inovar e setores industriais por CNAE 2.0. para o período de 2008

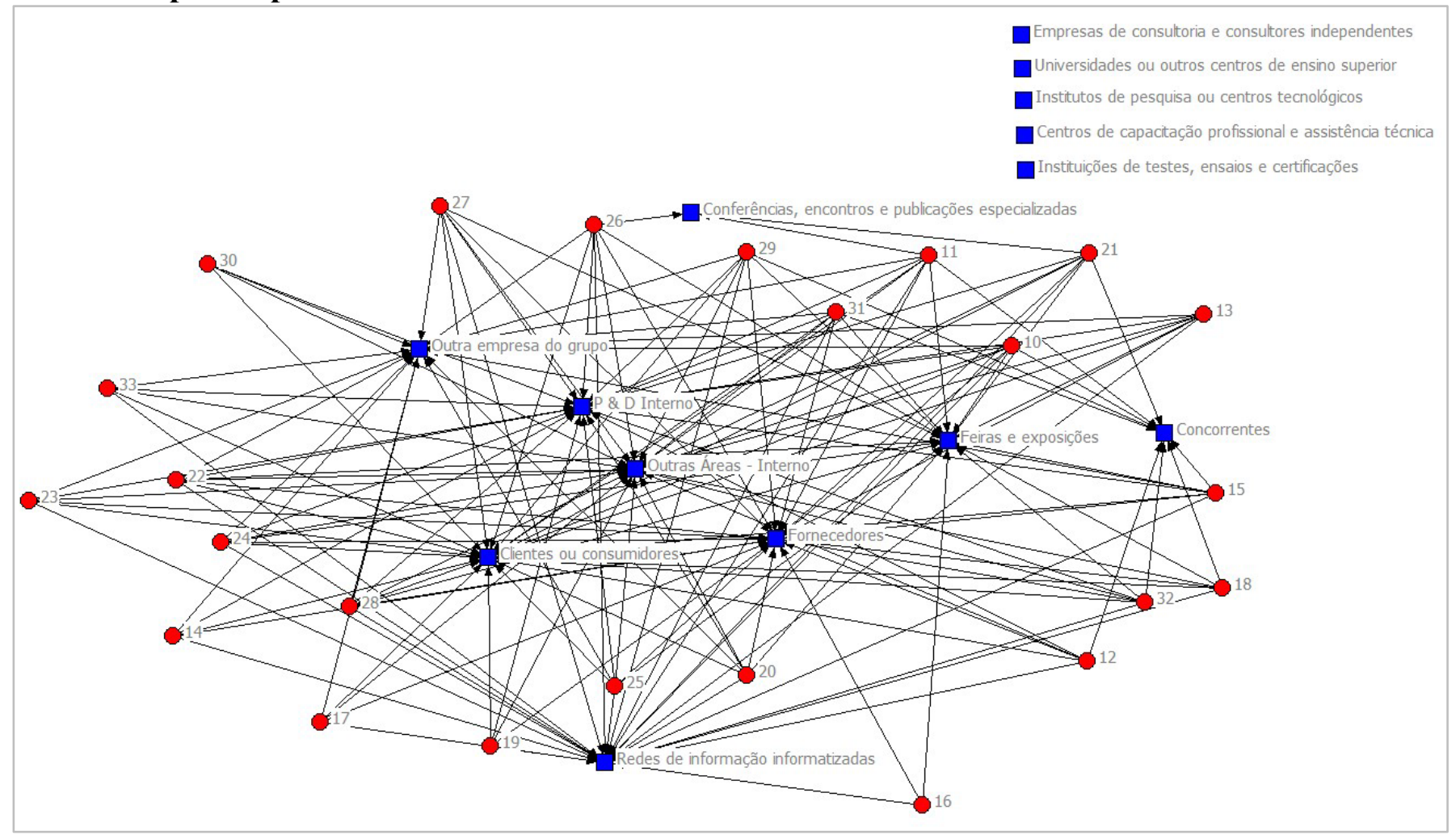

Fonte: Elaboração própria, a partir do Netdraw (BORGATTI, 2002). 
Figura 2: Estrutura dos Grafos das fontes de informação para inovar e setores industriais por CNAE 2.0. para o período de 2011

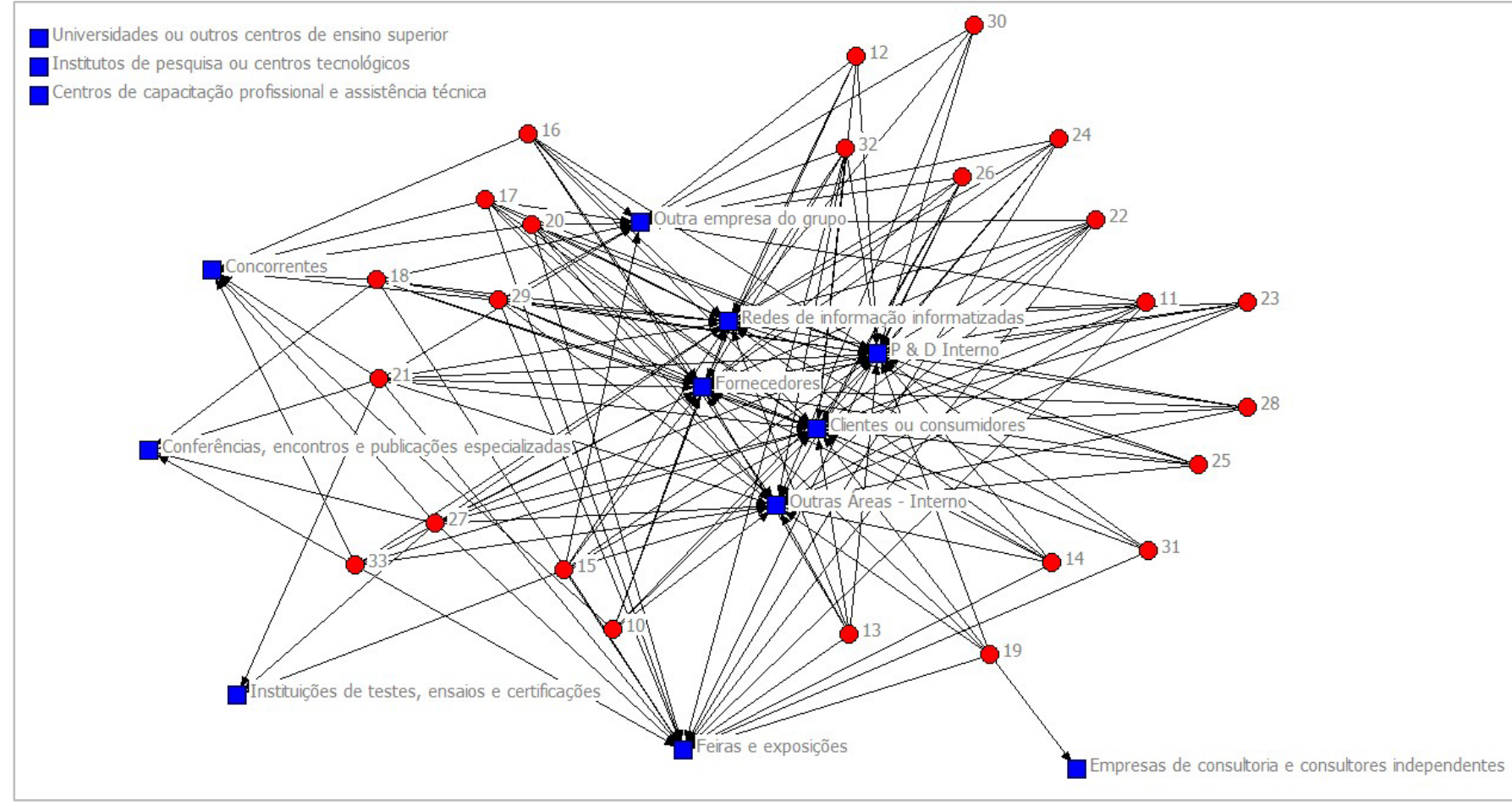

Fonte: Elaboração própria, a partir do Netdraw (BORGATTI, 2002).

Figura 3: Estrutura dos Grafos das fontes de informação para inovar e setores industriais por CNAE 2.0. para o período de 2014

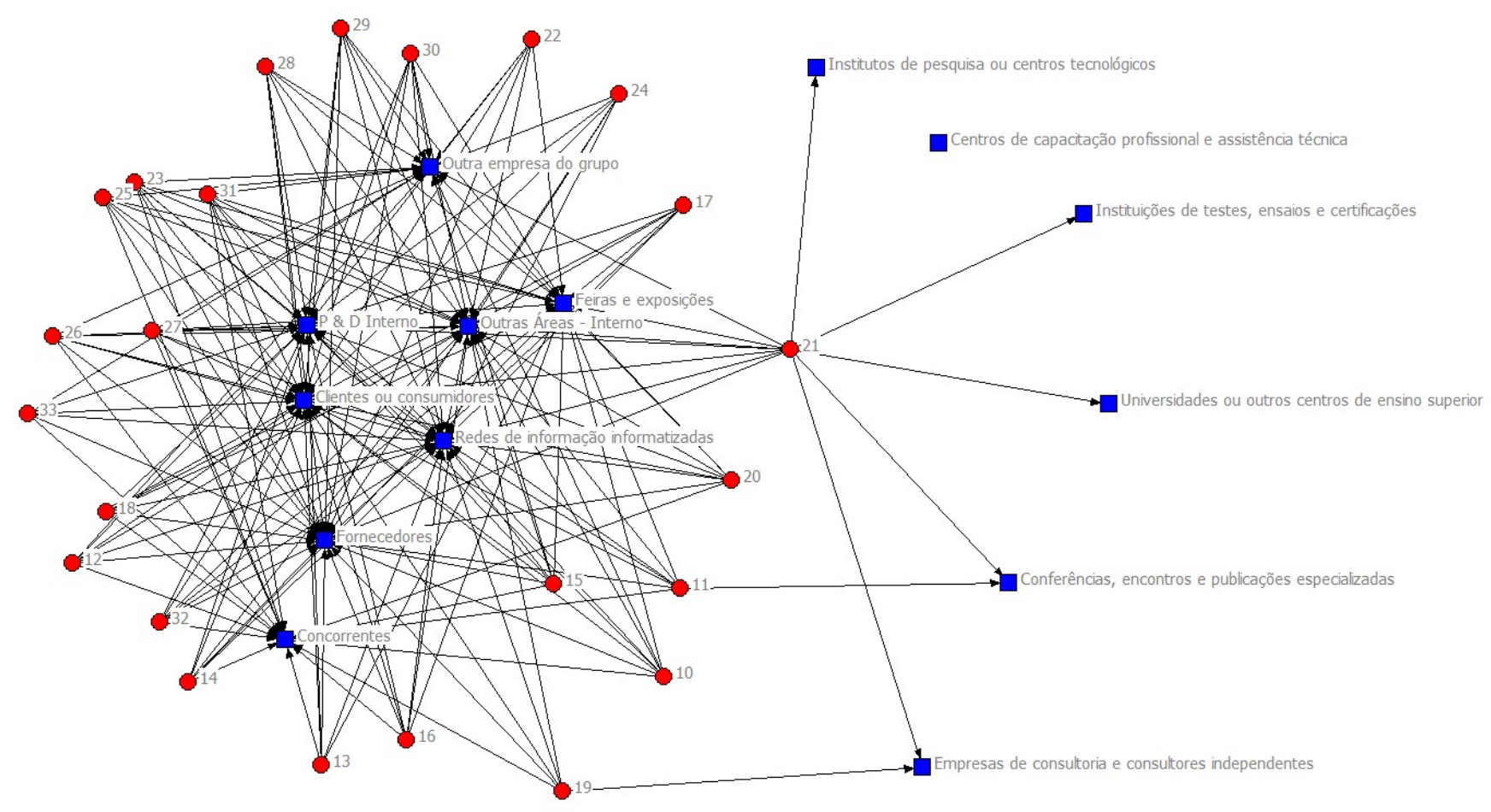

Fonte: Elaboração própria, a partir do Netdraw (BORGATTI, 2002).

Sob a perspectiva setorial, considerando os indicadores de centralidade em questão, levando em conta se a maioria das firmas de um setor considera relevante a respectiva fonte de informação para a atividade inovativa, os principais setores em relação à centralidade de grau são "Fabricação de produtos 
farmoquímicos e farmacêuticos", "Fabricação de bebidas", "Fabricação de produtos químicos", "Preparação de couros e fabricação de artefatos de couro, artigos para viagem" e "Fabricação de máquinas, aparelhos e materiais elétricos".

Com relação à centralidade de informação, os setores mais intensos na busca de informação para o processo inovativo são "Fabricação de produtos farmoquímicos e farmacêuticos", "Fabricação de produtos químicos", "Preparação de couros e fabricação de artefatos de couro, artigos para viagem", "Fabricação de produtos alimentícios" e "Impressão e reprodução de gravações".

Já para a centralidade de autovetor, os setores relevantes são "Fabricação de máquinas, aparelhos e materiais elétricos", "Fabricação de produtos alimentícios", "Fabricação de produtos químicos", "Preparação de couros e fabricação de artefatos de couro, artigos para viagem" e "Fabricação de produtos de minerais não-metálicos”.

Os setores industriais menos intensos na busca de fontes de informação para inovar, considerando as centralidades de grau, informação e autovetor, são "Fabricação de produtos de minerais não-metálicos", "Manutenção, reparação e instalação de máquinas e equipamentos", "Fabricação de produtos têxteis", "Metalurgia" e "Fabricação de celulose, papel e produtos de papel".

Quanto ao comportamento temporal das referidas centralidades dos setores industriais que possuem as menores centralidades são relativamente constantes nos períodos de 2008, 2011 e 2014, conforme tabela 1 disposta no apêndice.

Como a centralidade de grau denota o volume de atividade relacional, isto é, apresenta-se com maior notoriedade frente aos demais agentes, pode-se intuir que os setores industriais listados com maiores valores para a referida centralidade possuem elevada notoriedade frente aos demais setores, relacionado à diversificação na busca das fontes de informação para o processo inovativo.

Neste caso, segundo Packer (2018), podemos concluir, a partir da centralidade de grau que os setores que apresentam os menores valores, são setores industriais periféricos na rede no que diz respeito a busca pelas fontes de informação para inovação, nos períodos de 2008, 2011 e 2014.

Sobre a centralidade de informação, os setores industriais que apresentam os maiores valores referente a centralidade em questão, são setores capazes de receber informação do ambiente da rede construída, sendo os menores valores identificados como setores periféricos e menos diversificados na busca pela informação. Cabe salientar que tal centralidade é visto, neste caso, como uma complementariedade da centralidade de grau.

A relevância da centralidade de autovetor para a análise proposta se deve a importância de um setor industrial a medida de que busque acessar as fontes de informação para inovar, mantendo relações com agentes considerados relevantes na rede.

Como se buscou verificar a intensidade de busca por fontes de informação para o processo inovativo pelos setores industriais, a utilização da taxonomia proposta pela OECD (2011) e a sistematização da taxonomia de Pavitt (1984), expandido e aplicado sob a perspectiva setorial de acordo com Cavalcanti (2014) pela divisão dos setores industriais por intensidade tecnológica teve sua relevância para verificar quais setores industriais por intensidade tecnológica são mais ou menos assertivos na busca de fontes de informação para inovar.

Os resultados, comparados com a taxonomia proposta pela OECD, verifica-se certa heterogeneidade dos segmentos por intensidade tecnológica, sendo os setores de baixa e média-baixa intensidade tecnológica os mais pujantes na busca de informação para inovar, sendo 3 (três) dos 5 (cinco) setores nesta classificação, segundo as centralidades listadas.

Apenas um setor de alta tecnologia e um setor de média-alta tecnologia estão entre os principais segmentos na busca pela informação para inovar, sendo os demais segmentos industriais nesta mesma classificação estão em situações intermediárias. Os segmentos industriais menos intensos na busca pela informação para inovar são segmentos de média-baixa e baixa tecnologia.

Se observarmos a taxonomia de Pavitt (1984), adaptado para setores industriais por Dosi et al. (2008), pode-se verificar que setores intensivos em escala, seja processo contínuo ou descontínuo, são a maioria dos setores relevantes quanto a intensidade na busca de informações para inovar, possuindo ainda o setor de "Preparação de couros e fabricação de artefatos de couro, artigos para viagem", classificado como setor dominado por fornecedores, "Fabricação de máquinas, aparelhos e materiais elétricos", classificado 
como difusor de progresso técnico e "Fabricação de produtos farmoquímicos e farmacêuticos", classificado como baseado em ciência, dado a metodologia de cálculo de centralidade implementada neste trabalho.

Dentre os segmentos menos intensos na busca de informação, destacam-se segmentos classificados como intensivos em escala (processo contínuo), difusor de progresso técnico e dominado por fornecedores.

Chama atenção o comportamento descrito pelas centralidades calculadas para o setor de "Fabricação de equipamentos de informática, produtos eletrônicos e ópticos" e "Fabricação de máquinas e equipamentos", classificados respectivamente como setor baseado em ciência e difusor de progresso técnico, que estão em uma situação intermediária, porém próxima da situação periférica, considerando que tais setores deveriam apresentar uma dinâmica diferenciada na busca de informações para inovar.

Muito embora Johnson e Lundvall (2005) ressaltem que a economia do aprendizado não está limitado aos setores de elevado conteúdo tecnológico, tendo em vista que o processo de aprendizado está enraizado em toda camada produtiva e o fomento desta é relevante para a conformação de novas capacidades e habilidades que podem diferenciar as organizações de seus concorrentes, estes segmentos industriais possuem a capacidade de transbordamento de sua produtividade para os demais setores, além de impulsionar e direcionar a produção tecnológica em sua fronteira.

Assim, verificar que estes seguimentos de elevado conteúdo tecnológico na indústria brasileira estão em uma situação próxima à periferia da rede construída, denota que a intensidade pela busca de informações para implementar novos projetos tecnológicos estarão relacionados, de forma relativa, com o grau de especialização produtiva da indústria.

Quanto à relevância das fontes de informação para o processo inovativo, de acordo com a indicação dos setores da indústria de transformação brasileira, para o período de análise indicado, os que possuem as maiores centralidades indicadas são as "Redes de informação informatizadas", "Clientes ou consumidores" e "Fornecedores" - sendo as duas últimas fontes relacionadas com o learning by interacting -, conforme pode-se verificar na tabela 2 constante no apêndice.

A persistência das fontes de informação para inovar listadas como mais relevantes para os setores industriais ao longo do período de análise deste trabalho, evidencia, em certo grau, um padrão de concentração de fontes acessadas pelas organizações para subsidiar implementação de projetos inovadores, resultado semelhante ao encontrado em Bittencourt, Britto e Giglio (2016).

Segundo Malerba (1992) e Teece (2006), a heterogeneidade das fontes de informação para formação de novas competências e habilidades, é uma construção relevante no processo de aprendizagem para consolidação e ampliação do estoque de conhecimento necessário para a criação de novas tecnologias, para sua diferenciação no ambiente de competição.

Assim, a tendência verificada ao longo do tempo das principais fontes de inovação da indústria brasileira denota o aspecto incremental da construção de novas capacitações e habilidades em busca do novo. Mesmo considerando que uma das fontes relevantes são as redes de informação informatizadas, ao conectar as fontes utilizadas com os setores industriais mais intensos na busca de informação, percebe-se que são características intrínsecas de setores de baixa e média-baixa tecnologia, podendo se intuir que a direção adotada é a busca de tecnologias estabelecidas no mercado.

Em uma situação próxima às principais fontes de informação acessadas pelos segmentos industriais no Brasil, a fonte de informação relacionado com "P\&D Interno" e outras áreas da empresa que não estejam ligadas com a área de P\&D interno, conforme tabela 2. Com relação ao "P\&D Interno", evidencia uma relativa importância do learning by searching e para outras áreas da empresa que não estejam ligadas com a área de P\&D interno (CHANG; TZENG, 2010), reforça a presença relativa dos tipos de aprendizagem learning by doing e learning by using, resultado semelhante ao encontrado em Bittencourt (2012) e Bittencourt, Britto e Giglio (2016).

Cabe salientar que as fontes de informação como "Feiras e exposições" e "Concorrentes", com uma posição intermediária quanto à relevância para o processo inovativo de acordo com as medidas de centralidade listadas neste trabalho, expõem uma característica relevante do processo de aprendizagem da indústria brasileira, chamado por Malerba (1992) como learning from interindustry spillovers, sendo o processo de aprendizado associado a capacidade de imitação das organizações.

Quanto às fontes de informação menos relevantes para auxiliar na conformação dos projetos de inovação, as fontes de informação que apresentaram valores baixos de acordo com as medidas de 
centralidade são "Institutos de pesquisa ou centros tecnológicos", "Universidades ou outros centros de ensino superior", "Instituições de testes, ensaios e certificações" e "Centros de capacitação profissional e assistência técnica".

Chama atenção os resultados apresentados para os institutos de pesquisa ou centros tecnológicos, universidades ou outros centros de ensino superior, considerando os resultados apresentados pela literatura sobre os tipos de informação listados. Britto e Oliveira (2011) e Del-Vecchio, Britto e Oliveira (2014) ressaltam a importância da interação entre a universidade e a indústria como forma de geração de novas capacitações em busca de soluções relevantes para sociedade.

As fontes de informação advindas de universidades e institutos de pesquisa estão relacionadas com a produção científica e a sua interação com o setor industrial é relevante para transformar essa produção científica em produtos e/ou processos de produção que alterem as condições competitivas das organizações, além de propiciar ganhos de bem-estar para a sociedade. Verificar a baixa centralidade dessas fontes de informação para a indústria brasileira, bem como a sua persistência ao longo dos períodos de 2008, 2011 e 2014, denota a fragilidade do sistema nacional de inovação, bem como a baixa capacidade de agregação de novos conhecimentos que estão na fronteira tecnológica.

Se observarmos a figura 1, percebe-se que fica evidente que o único setor que se conecta com a universidade, "Centros de capacitação profissional e assistência técnica", "instituições de teste, ensaios e certificações" e "Conferências, encontros e publicações especializadas" é o setor de "Fabricação de produtos farmoquímicos e farmacêuticos", relação estabelecida somente na pesquisa de 2014, evidenciando a concentração das fortes de informação da indústria brasileira no caráter incremental, não se conectando com fontes que podem impulsionar para a busca de novas tecnologias que possam ser cruciais para a diferenciação de seus concorrentes e elevar a capacidade competitiva no mercado internacional, podendo impactar na limitação da dinâmica inovativa dos setores industriais (BITTENCOURT; BRITTO; GIGLIO, 2016).

\section{Considerações Finais}

O objetivo deste artigo foi mapear por meio da análise das medidas de centralidade de grafos a estrutura de fontes de informação para o processo inovativo da indústria brasileira, listando os setores industriais mais relevantes na busca das referidas fontes de informação, bem como mensurar quais fontes de informação são mais relevantes para o processo inovativo da indústria brasileira.

Para viabilizar a análise, foram utilizados os dados das empresas que implementaram inovações, com a consideração do grau de importância listada pela PINTEC, elaborada pelo IBGE, para os períodos de 2008, 2011 e 2014, adotando como metodologia uma relação binária, considerando se a maioria das empresas consideram tal informação relevante para o processo inovativo.

A partir das medidas de centralidade de grau, informação e de autovetor, os resultados encontrados para os setores industriais evidenciam certa heterogeneidade da busca por setor industrial, segregado por intensidade tecnológica.

Porém, verifica-se que os segmentos que buscam de forma mais intensa as fontes de informação para construção de capacidades para implementar inovações são os setores de baixa e média-baixa intensidade tecnológica, estando os setores de média-alta e alta intensidade tecnológica, dependendo da medida de centralidade adotada, em níveis intermediários e segmentos de baixa e média-baixa intensidade tecnológica com pouca intensidade na busca por fontes de informação pra inovação.

Quanto a relevância das fontes de informação, considerando as medidas de centralidade de grafos utilizadas, verifica-se que ao longo do período de análise um padrão de concentração das fontes utilizadas pelas empresas, relacionadas com os clientes ou consumidores, redes de informações informatizadas, fornecedores, feiras, P\&D interno, exposições e concorrentes, evidenciando o aspecto incremental e imitativo dos projetos de inovação da indústria.

Com este trabalho, pode-se verificar a baixa representatividade das universidades, institutos de pesquisa como fontes de informação relevante para implementação das inovações por parte da indústria brasileira, reforçando as evidências encontradas relacionadas com o aspecto incremental e imitativo dos projetos de inovação da indústria. 
Embora o desenvolvimento desta pesquisa possua limitações, os resultados encontrados propiciam uma janela de novas investigações sobre o tema, como a relação entre as fontes de informações para inovar e o estabelecimento de cooperação e parcerias, para identificar o comportamento do processo de aprendizado e interação dos agentes, relacionados à indústria brasileira, de forma a subsidiar políticas públicas de fortalecimento dos sistema nacional, regional ou setorial de inovação.

Além disso, faz-se necessário uma investigação com um período mais longo do aspecto de aprendizagem da indústria, relacionando também com as barreiras à implementação de projetos inovativos por parte das organizações.

\section{Referências}

BITTENCOURT, Pablo Felipe. Padrões setoriais de aprendizagem da indústria brasileira: uma análise exploratória. Revista Brasileira de Inovação, v. 11, n. 1, p. 37-68, 2012.

BITTENCOURT, Pablo Felipe; BRITTO, Jorge Nogueira de Paiva; GIGLIO, Ricardo. Formas de aprendizagem e graus de inovação de produto no Brasil: uma análise exploratória dos padrões setoriais de aprendizagem. Nova Economia, v. 26, n. 1, 2016.

BONACICH, Phillip. Power and centrality: A family of measures. American journal of sociology, v. 92, n. 5, p. 1170-1182, 1987.

BORGATTI, S.P. 2002. NetDraw: Graph Visualization Software. Harvard: Analytic Technologies

BORGATTI, S.P., EVERETT, M.G. and FREEMAN, L.C. 2002. Ucinet 6 for Windows: Software for Social Network Analysis. Harvard, MA: Analytic Technologies.

BORGATTI, Stephen P. Centrality and network flow. Social networks, v. 27, n. 1, p. 55-71, 2005.

BORGATTI, Stephen P.; EVERETT, Martin G. A graph-theoretic perspective on centrality. Social networks, v. 28, n. 4, p. 466-484, 2006.

BRITTO, Jorge; OLIVEIRA, Bruno Ferreira de. Padrões setoriais de interação universidade-empresa no Brasil: um mapeamento de competências a partir de informações da "Brazil Survey". Revista de Economia, v. 37, n. 4, 2011.

CAMPOS, Bruno; RUIZ, Ana Urraca. Padrões setoriais de inovação na indústria brasileira. Revista Brasileira de Inovação, v. 8, n. 1, p. 167-210, 2009.

CAVALCANTE, Luiz Ricardo. Classificações tecnológicas: uma sistematização. 2014.

CHANG, Hung-Fan; TZENG, Gwo-Hshiung. A causal decision making model for knowledge management capabilities to innovation performance in taiwan's high-tech industry. Journal of technology management \& innovation, v. 5, n. 4, p. 137-146, 2010.

DE FREITAS, Leandro Quintanilha. Medidas de centralidade em grafos. 2010. Tese de Doutorado. dissertação de mestrado, Universidade Federal do Rio de Janeiro.

DEL-VECCHIO, Renata R.; BRITTO, Jorge; OLIVEIRA, Bruno F de. Patterns of university-industry interactions in Brazil: an exploratory analysis using the instrumental of graph theory. Quality \& Quantity, v. 48, n. 4, p. 1867-1892, 2014. 
DOSI, Giovanni et al. Technological revolutions and the evolution of industrial structures: assessing the impact of new technologies upon the size and boundaries of firms. Capitalism and Society, v. 3, n. 1, 2008.

DOSI, Giovanni. Mudança técnica e transformação industrial: a teoria e uma aplicação à indústria dos semicondutores. Editora Unicamp, 2006.

DOSI, Giovanni; MALERBA, Franco; ORSENIGO, Luigi. Evolutionary regimes and industrial dynamics. In: Evolutionary and neo-Schumpeterian approaches to economics. Springer, Dordrecht, 1994. p. $203-$ 229.

EDQUIST, Charles; LUNDVALL, Bengt-Ake. Comparing the Danish and Swedish systems of innovation. National innovation systems: A comparative analysis, p. 265-298, 1993.

FERRAZ, João Carlos; KUPFER, David; HAGUENAUER, Lia. Made in Brazil: desafios competitivos para a indústria. Rio de janeiro: Campus, p. 386, 1995.

FERREIRA, André Luiz Bispo et al. Redes sociais: um estudo introdutório. 2014.

FREEMAN, Chris. The 'National System of Innovation' in historical perspective. Cambridge Journal of economics, v. 19, n. 1, p. 5-24, 1995.

FREEMAN, Christopher. Technology policy and economic performance/C. London, NY, 1987.

FREEMAN, Linton C. Centrality in social networks conceptual clarification. Social networks, v. 1, n. 3, p. 215-239, 1978.

GONCALVES, Eduardo; SIMÕES, Rodrigo. Padrões de esforço tecnológico da indústria brasileira: uma análise setorial a partir de técnicas multivariadas. Revista EconomiA, 2005.

HURTADO, Rafael Germán; MEJÍA, Jorge Enrique. Estructura de la inversión de la industria manufacturera colombiana en actividades de innovación y desarrollo tecnológico. Innovar: Revista de ciencias administrativas y sociales, p. 33-40, 2014.

INSTITUTO BRASILEIRO DE GEOGRAFIA E ESTATÍSTICA (IBGE). Rio de Janeiro, 2016. Disponível em http://www.ibge.gov.br. Acesso em 20/01/2019.

JOHNSON, Bjørn Harold; LUNDVALL, Bengt-Åke. Promovendo sistemas de inovação como resposta à economia do aprendizado crescentemente globalizada. In: Conhecimento, sistemas de inovação e desenvolvimento. Editora UFRJ, 2005. p. 83-130.

KOENIG, Michael D.; BATTISTON, Stefano. From graph theory to models of economic networks. a tutorial. Networks, topology and dynamics, v. 613, p. 23-63, 2009.

LAZONICK, William. Understanding innovative enterprise: Toward the integration of economic theory and business history. manuscript, University of Masachusetss Lowell and The European Institute of Business Administration (INSEAD), Fontainebleau, May, 2000.

LASTRES, Helena Maria Martins; FERRAZ, João Carlos. Economia da informação, do conhecimento e do aprendizado. Informação e globalização na era do conhecimento. Rio de Janeiro: Campus, p. 2757, 1999. 
LUNDVALL, Bengt-Ake. National systems of innovation: An analytical framework. London: Pinter, 1992.

MALERBA, Franco (Ed.). Sectoral systems of innovation: concepts, issues and analyses of six major sectors in Europe. Cambridge University Press, 2004.

MALERBA, Franco. Learning by firms and incremental technical change. The economic journal, v. 102, n. 413 , p. $845-859,1992$.

MALERBA, Franco. Sectoral systems of innovation and production. Research policy, v. 31, n. 2, p. 247264, 2002.

METCALFE, J. Stan. Technology systems and technology policy in an evolutionary framework. Cambridge journal of economics, v. 19, n. 1, p. 25-46, 1995.

PACKER, Osni. Posições de poder e influência nos fluxos de relações em uma agência de desenvolvimento regional do estado de Santa Catarina. 2018.

PAVITT, Keith. Sectoral patterns of technical change: towards a taxonomy and a theory. Research policy, v. 13, n. 6 , p. 343-373, 1984.

REV, OECD ISIC. technology intensity definition. 2011. Classification of Manufacturing Industries into Categories Based on R\&D Intensities, 3.

SILVA, Conceição de Fátima; SUZIGAN, Wilson. Padrões setoriais de inovação da indústria de transformação brasileira. Estudos Econômicos (São Paulo), v. 44, n. 2, p. 277-321, 2014.

TEECE, David J. As aptidões das empresas e o desenvolvimento econômico: implicações para as economias de industrialização recente. Tecnologia, aprendizado e inovação: as experiências das economias de industrialização recente, 2005.

TEECE, David J.; PISANO, Gary; SHUEN, Amy. Dynamic capabilities and strategic management. Strategic management journal, v. 18, n. 7, p. 509-533, 1997.

WILSON, R. J. Introduction to Graph Theory. Fourth Edition. Prentice-hall, 1996 


\section{APÊNDICE}

Tabela 1: Cálculo das Centralidades de grau, informação e autovetor para a indústria brasileira, em termos de ordenamento (continua).

\begin{tabular}{|c|c|c|c|c|c|c|c|c|c|c|c|}
\hline \multirow[t]{2}{*}{ Descrição dos Setores Industriais } & \multicolumn{3}{|c|}{$\begin{array}{l}\text { Centralidade de Grau } \\
\text { (Normalizado) }\end{array}$} & \multicolumn{3}{|c|}{$\begin{array}{l}\text { Centralidade de } \\
\text { Informação }\end{array}$} & \multicolumn{3}{|c|}{$\begin{array}{l}\text { Centralidade de } \\
\text { Autovetor }\end{array}$} & \multirow{2}{*}{$\begin{array}{l}\text { Classificação } \\
\text { OECD }\end{array}$} & \multirow{2}{*}{$\begin{array}{c}\text { Classificação } \\
\text { Pavitt }\end{array}$} \\
\hline & 2008 & 2011 & 2014 & 2008 & 2011 & 2014 & 2008 & 2011 & 2014 & & \\
\hline Fabricação de produtos alimentícios & 2 & 14 & 2 & 1 & 14 & 2 & 2 & 16 & 1 & Baixa & IEd \\
\hline Fabricação de bebidas & 1 & 8 & 2 & 1 & 3 & 8 & 1 & 7 & 8 & Baixa & IEc \\
\hline Fabricação de produtos do fumo & 16 & 19 & 9 & 2 & 18 & 17 & 17 & 23 & 17 & Baixa & IEd \\
\hline Fabricação de produtos têxteis & 7 & 14 & 20 & 2 & 14 & 22 & 6 & 13 & 22 & Baixa & $\mathrm{DF}$ \\
\hline $\begin{array}{l}\text { Confecção de artigos do vestuário e } \\
\text { acessórios }\end{array}$ & 19 & 14 & 9 & 2 & 14 & 9 & 20 & 13 & 9 & Baixa & DF \\
\hline $\begin{array}{l}\text { Preparação de couros e fabricação de } \\
\text { artefatos de couro, artigos para viagem }\end{array}$ & 7 & 3 & 2 & 2 & 3 & 2 & 15 & 5 & 1 & Baixa & $\mathrm{DF}$ \\
\hline Fabricação de produtos de madeira & 24 & 8 & 9 & 2 & 3 & 9 & 24 & 12 & 9 & Baixa & $\mathrm{DF}$ \\
\hline $\begin{array}{l}\text { Fabricação de celulose, papel e } \\
\text { produtos de papel }\end{array}$ & 19 & 3 & 20 & 2 & 3 & 20 & 21 & 2 & 20 & Baixa & DF \\
\hline Impressão e reprodução de gravações & 16 & 3 & 9 & 2 & 3 & 9 & 18 & 11 & 9 & Baixa & $\mathrm{DF}$ \\
\hline $\begin{array}{l}\text { Fabricação de coque, de produtos } \\
\text { derivados do petróleo e de } \\
\text { biocombustíveis }\end{array}$ & 19 & 14 & 20 & 2 & 18 & 24 & 19 & 22 & 24 & Média-Baixa & IEc \\
\hline Fabricação de produtos químicos & 7 & 3 & 2 & 2 & 3 & 2 & 6 & 2 & 1 & Média-Alta & IEc \\
\hline $\begin{array}{l}\text { Fabricação de produtos } \\
\text { farmoquímicos e farmacêuticos }\end{array}$ & 2 & 1 & 1 & 1 & 1 & 1 & 13 & 1 & 7 & Alta & $\mathrm{BC}$ \\
\hline $\begin{array}{l}\text { Fabricação de produtos de borracha e } \\
\text { de material plástico }\end{array}$ & 7 & 8 & 9 & 2 & 3 & 9 & 6 & 7 & 13 & Média-Baixa & DF \\
\hline $\begin{array}{l}\text { Fabricação de produtos de minerais } \\
\text { não-metálicos }\end{array}$ & 16 & 19 & 2 & 2 & 18 & 2 & 16 & 17 & 1 & Média-Baixa & IEd \\
\hline Metalurgia & 7 & 14 & 20 & 2 & 14 & 21 & 6 & 15 & 21 & Média-Baixa & IEc \\
\hline $\begin{array}{l}\text { Fabricação de produtos de metal, } \\
\text { exceto máquinas e equipamentos }\end{array}$ & 7 & 19 & 9 & 2 & 18 & 17 & 6 & 17 & 19 & Média-Baixa & IEd \\
\hline
\end{tabular}


Tabela 1: Cálculo das Centralidades de grau, informação e autovetor para a indústria brasileira, em termos de ordenamento (continuação).

\begin{tabular}{|c|c|c|c|c|c|c|c|c|c|c|c|}
\hline \multirow[t]{2}{*}{ Descrição dos Setores Industriais } & \multicolumn{3}{|c|}{$\begin{array}{l}\text { Centralidade de Grau } \\
\text { (Normalizado) }\end{array}$} & \multicolumn{3}{|c|}{$\begin{array}{l}\text { Centralidade de } \\
\text { Informação }\end{array}$} & \multicolumn{3}{|c|}{$\begin{array}{l}\text { Centralidade de } \\
\text { Autovetor }\end{array}$} & \multirow{2}{*}{$\begin{array}{l}\text { Classificaçãa } \\
\text { OECD }\end{array}$} & \multirow{2}{*}{$\begin{array}{c}\text { Classificação } \\
\text { Pavitt }\end{array}$} \\
\hline & 2008 & 2011 & 2014 & 2008 & 2011 & 2014 & 2008 & 2011 & 2014 & & \\
\hline $\begin{array}{l}\text { Fabricação de equipamentos de } \\
\text { informática, produtos eletrônicos e } \\
\text { ópticos }\end{array}$ & 2 & 8 & 9 & 1 & 3 & 17 & 5 & 7 & 17 & Alta & $\mathrm{BC}$ \\
\hline $\begin{array}{l}\text { Fabricação de máquinas, aparelhos e } \\
\text { materiais elétricos }\end{array}$ & 7 & 2 & 2 & 2 & 1 & 2 & 6 & 5 & 1 & Média-Alta & DPT \\
\hline $\begin{array}{l}\text { Fabricação de máquinas e } \\
\text { equipamentos }\end{array}$ & 7 & 19 & 9 & 2 & 18 & 9 & 6 & 17 & 13 & Média-Alta & DPT \\
\hline $\begin{array}{l}\text { Fabricação de veículos automotores, } \\
\text { reboques e carrocerias }\end{array}$ & 2 & 3 & 9 & 1 & 3 & 9 & 2 & 2 & 13 & Média-Alta & IEd \\
\hline $\begin{array}{l}\text { Fabricação de outros equipamentos de } \\
\text { transporte, exceto veículos } \\
\text { automotores }\end{array}$ & 23 & 24 & 9 & 2 & 24 & 9 & 23 & 24 & 13 & Média-Alta & IEd \\
\hline Fabricação de móveis & 2 & 19 & 2 & 1 & 18 & 2 & 2 & 21 & 1 & Baixa & $\mathrm{DF}$ \\
\hline Fabricação de produtos diversos & 7 & 8 & 9 & 2 & 3 & 9 & 14 & 7 & 9 & Baixa & DF \\
\hline $\begin{array}{l}\text { Manutenção, reparação e instalação de } \\
\text { máquinas e equipamentos }\end{array}$ & 19 & 8 & 20 & 2 & 3 & 23 & 22 & 17 & 23 & Média-Baixa & DPT \\
\hline
\end{tabular}

Fonte: Elaboração própria a partir dos dados do IBGE (2016), OECD (2011) e Calvacante (2014). 
Tabela 2: Cálculo das Centralidades de grau, informação e autovetor para as fontes de informação para inovação da indústria brasileira em termos de ordenamento

\begin{tabular}{|c|c|c|c|c|c|c|c|c|c|}
\hline \multirow[t]{2}{*}{ Descrição das fontes de informção } & \multicolumn{3}{|c|}{$\begin{array}{l}\text { Centralidade de Grau } \\
\text { (Normalizado) }\end{array}$} & \multicolumn{3}{|c|}{$\begin{array}{l}\text { Centralidade de } \\
\text { Informação }\end{array}$} & \multicolumn{3}{|c|}{$\begin{array}{l}\text { Centralidade de } \\
\text { Autovetor }\end{array}$} \\
\hline & 2008 & 2011 & 2014 & 2008 & 2011 & 2014 & 2008 & 2011 & 2014 \\
\hline P \& D Interno & 4 & 1 & 4 & 1 & 1 & 5 & 4 & 4 & 4 \\
\hline Outras Áreas - Interno & 4 & 5 & 4 & 1 & 1 & 4 & 5 & 5 & 4 \\
\hline Outra empresa do grupo & 6 & 7 & 8 & 1 & 6 & 8 & 6 & 7 & 8 \\
\hline Fornecedores & 3 & 1 & 1 & 1 & 1 & 1 & 3 & 1 & 1 \\
\hline Clientes ou consumidores & 1 & 1 & 1 & 1 & 1 & 1 & 1 & 1 & 1 \\
\hline Concorrentes & 8 & 8 & 7 & 1 & 8 & 7 & 8 & 8 & 7 \\
\hline $\begin{array}{l}\text { Empresas de consultoria e consultores } \\
\text { independentes }\end{array}$ & 10 & 11 & 9 & 10 & 11 & 10 & 10 & 11 & 10 \\
\hline $\begin{array}{l}\text { Universidades ou outros centros de } \\
\text { ensino superior }\end{array}$ & 10 & 11 & 11 & 11 & 12 & 11 & 10 & 12 & 11 \\
\hline $\begin{array}{l}\text { Institutos de pesquisa ou centros } \\
\text { tecnológicos }\end{array}$ & 10 & 11 & 11 & 11 & 13 & 11 & 10 & 12 & 11 \\
\hline $\begin{array}{l}\text { Centros de capacitação profissional e } \\
\text { assistência técnica }\end{array}$ & 10 & 11 & 14 & 11 & 13 & 14 & 10 & 12 & 14 \\
\hline $\begin{array}{l}\text { Instituições de testes, ensaios e } \\
\text { certificações }\end{array}$ & 10 & 10 & 11 & 11 & 10 & 11 & 10 & 10 & 11 \\
\hline $\begin{array}{l}\text { Conferências, encontros e publicações } \\
\text { especializadas }\end{array}$ & 9 & 9 & 9 & 9 & 9 & 9 & 9 & 9 & 9 \\
\hline Feiras e exposições & 7 & 6 & 6 & 1 & 6 & 6 & 7 & 6 & 6 \\
\hline Redes de informação informatizadas & 1 & 1 & 1 & 1 & 1 & 1 & 2 & 3 & 1 \\
\hline
\end{tabular}

Fonte: Elaboração própria a partir dos dados do IBGE (2016). 